\title{
Violencia y victimización entre escolares. El bullying: estrategias de identificación y elementos para la intervención a través del Test Bull-S
}

\section{Fuensanta Cerezo}

\author{
Dpto. de Psicología Evolutiva y de la Educación, \\ Universidad de Murcia
}

España

fcerezo@um.es 
Violencia y victimización entre iguales. El bullying: estrategias de identificación y elementos para la intervención a través del Test Bull-S

\section{Resumen}

Introducción. El Informe del Defensor del Pueblo sobre violencia escolar-bullying- puso de manifiesto que en la mayoría de los centros educativos surgen situaciones de abuso y violencia entre los escolares (AA.VV., 2000). El contacto con profesionales de la enseñanza general nos revela que, con frecuencia, este fenómeno sólo llega a conocimiento de la comunidad educativa cuando, por desgracia, suele ser demasiado tarde, es decir, cuando las conductas de agresión y de victimización están muy arraigadas y su repercusión es dramática. Numerosos estudios sitúan el bullying como una manifestación de las malas relaciones interpersonales entre los alumnos (Ortega, 1994; Sutton y Smith, 1999). Ahondando en esta cuestión nos planteamos la influencia que puede tener la red de relaciones que se generan en el grupo aula y hasta que punto el víctima lo es no sólo de los ataques de su agresor sino del clima social que vive el grupo, que como un sistema cerrado lo excluye, mientras que el agresor cobra relevancia (Cerezo, Calvo y Sánchez, 2004).

Objetivos. Desde las primeras investigaciones buscamos técnicas que permitieran la detección temprana del bullying, y que, a su vez, sirvieran de base para elaborar programas de intervención. Nos planteamos el diseño del test Bull como un instrumento que informara sobre la realidad social y afectiva del grupo aula y la implicación de sus miembros en el bullying, así como sobre características de tipo personal, de interacción social y escolar de los alumnos de cada grupo aula. El Bull-S. Test de evaluación de la agresividad entre escolares (Cerezo 2000), se presenta como una herramienta válida para la detección, medida y valoración de las situaciones de agresividad entre escolares. La prueba persigue tres objetivos fundamentales: Facilitar el análisis de las características socio-afectivas del grupo de iguales, ayudar al profesorado en la detección de situaciones de abuso entre escolares, y a través de sus resultados, avanzar en la elaboración de propuestas de intervención.

Método. El instrumento, siguiendo la línea metodológica de la Sociometría y a través de la técnica de peer nomination, analiza de la estructura interna del aula definida bajo los criterios: aceptación-rechazo, agresividad-victimización y la apreciación de determinadas características personales que se pueden asociar a los alumnos directamente implicados. 
Resultados y conclusiones. El trabajo que aqui se presenta resume las características de este instrumento y su aplicabilidad e incluye una propuesta de intervención para un grupo de alumnos elaborada a partir del análisis del test Bull-S

Palabras Clave: Bullying. Evaluación del bullying. Estrategias de intervención.

\section{Introducción}

La experiencia nos dice que la violencia interpersonal entre los escolares es un hecho y, según parece, disponemos de todos los ingredientes para justificarlo: "esto ha pasado siempre"; "no es tan grave que los chavales se peleen"; "tienen que aprender a defenderse". Además, el entorno social nos presenta la violencia como una forma de comportamiento valorada y atractiva, solo hay que ver el contenido de los juegos más vendidos o de las películas más taquilleras, por no hablar de ciertos programas de televisión. Las situaciones de violencia entre los escolares van más allá de los episodios concretos y puntuales de agresión y victimización. Estas situaciones evidencian un desequilibrio de fuerzas prolongado que se resuelve de manera no socializada, de manera que la víctima recibe las agresiones de otro de manera sistemática y llega a convertirse en su víctima habitual. La posición de indefensión es altamente contaminante de la percepción de victimización, de manera que suele extenderse al conjunto del grupo de iguales, hasta el punto de percibir que el ambiente escolar se vuelve contra él, lo que propicia el desarrollo de graves estados de ansiedad. Por otro lado, el agresor va afianzando su conducta antisocial, cuyas consecuencias provocan exclusión social y favorecen la predelincuencia.

El bullying, término acuñado por Dan Olweus (1993) para definir una forma específica de maltrato entre escolares, se caracteriza por ser intencionado y persistente de un alumno o grupo de ellos hacia otro alumno sin que medie provocación ni posibilidad de respuesta. Estos agresores o bullies suelen actuar movidos por el deseo de poder, de intimidar, dominar, aunque en ocasiones reconocen estar motivados por simple diversión. En esta diada la víctima está indefensa, sin posibilidad de respuesta ni apoyos. Las agresiones no necesariamente son físicas, quizá las de éste tipo sean las menos frecuentes, especialmente en edades de la Educación Secundaria (se aprecia entre el 7\% y el 5\%). Las agresiones verbales son mucho más comunes, desde el insulto, las amenazas, el correr rumores que tratan de mermar la estima, 
etc. (por encima del 30\% de las agresiones) e incluso la exclusión social y el aislamiento (situada en torno al $20 \%$ ), en mucho menor grado se aprecian las agresiones que atentan contra la propiedad (7\%) o el acoso sexual (2\%) (AA.VV, 2000).

Entre los factores que inciden en el desarrollo de estas conductas destaca el modelo social que proporcionan los adultos en los medios familiar y escolar, donde, sin duda, el mayor exponente lo conforman las experiencias de maltrato, sufrido u observado, como señala Barudy (1998). Pero es necesario un escenario propicio para que el bullying se manifieste y éste, es el grupo-aula. Dentro de los grupos de iguales juega un papel trascendente las relaciones interpersonales que conforman los roles y estatus a cada uno de sus miembros, los grupos se estructuran en torno a determinados componentes afectivos y relacionales que actúan como elementos básicos en la asignación de determinadas pautas comportamentales (Roland y Galloway, 2002). Si entendemos que el bullying es un fenómeno grupal, para comprender su alcance se hace imprescindible el análisis de las relaciones sociales entre los escolares.

Estos planteamientos sugieren que la repercusión de la victimización trasciende a la situación concreta y llega a modular incluso la percepción de la conducta de los no implicados, de manera que, cuando un sujeto recibe las agresiones de otro de manera sistemática, llega a ser víctima no sólo de los ataques de su agresor, sino de todo el entorno social en que vive (Gifford-Smith y Brownell, 2003). De igual modo, el agresor se encuentra en continuo estado de alerta. Los estudios vienen a confirmar que los alumnos agresores tienen mayor ascendencia social y, por tanto, son mejor considerados por al menos una parte importante de sus compañeros, mientras que a los sujetos víctimas se les atribuyen aspectos que, en cierta medida, favorecen el que se encuentren es esas situaciones de indefensión, ya que se les aísla de juegos y actividades. Estudios previos nos han llevado a señalar aspectos como la ascendencia social y en nivel de relaciones sociales en la génesis y mantenimiento de conductas bullying (Cerezo, 2001b; Cerezo, Calvo y Sánchez, 2004).

El Informe del Defensor del pueblo (AA.VV. 2000) destacaba que el bullying se aprecia en más del $50 \%$ de los centros escolares y en más de la mitad de los alumnos. Estudios recientes confirman que en la práctica totalidad de los centros educativos se aprecian situaciones de maltrato entre escolares; que entre el 25 y el 40 por ciento del alumnado está implicado (los chicos en mayor medida que las chicas) y especialmente en los últimos cursos de Primaria y los primeros de Secundaria. Resulta inquietante que los adultos, en general, continúan 
poco informados y asimismo que las víctimas sufren el maltrato de manera continuada y sin apenas hacer partícipe de su situación a nadie (Cerezo, 2005). Ciertamente, por un lado, los agresores y los "espectadores" se encargan de que los hechos no lleguen a ser conocidos por los profesores ni los padres, y por otro, los que sufren los ataques generan tal punto de indefensión, vergüenza y miedo, que difícilmente lo contarían a alguien, aunque tampoco sabrían bien a quién, porque, como hemos comentado, se sienten amenazados por el conjunto de sus compañeros de clase.

Así pues, estamos ante un problema donde confluyen las dos caras de la moneda, una que resulta fortalecida (el agresor) y otra que progresivamente va entrando en una espiral de exclusión (la víctima) (Leary, Kowalski, Smith y Phillis, 2003). Hasta tal punto los comportamientos son percibidos como diferentes que podríamos avanzar un perfil psicológico específico asociado. Es probable que este planteamiento resulte excesivamente simplista, si tenemos en cuenta que los motivos que llevan a estar en un lado o en otro pueden ser bien diferentes entre un sujeto y otro, e incluso, en ocasiones hay sujetos que participan de ambos perfiles, los llamados víctimas provocadores (Olweus, 1993)- que, generalmente siendo objeto de maltrato por algunos compañeros optan por victimizar a otros como respuesta a la situación de indefensión (Salmivalli y Nieminen, 2002), aunque puede ser de utilidad a la hora de buscar una categorización general (Cerezo, 1997).

Por tanto, para la evaluación del bullying, entendemos que no estamos ante un fenómeno de características similares para todos los sujetos implicados, sino que, se trata, al menos de dos tipos de problemáticas bien diferentes: por un lado la evaluación de los principales implicados, el actor de las agresiones o bully y de otro lado el receptor de las mismas o víctima (aunque, en ocasiones puede darse que un mismo alumno participe de ambos roles); y paralelamente se debe valorar hasta qué punto el resto del grupo, los observadores, aceptan, apoyan o sancionan estas actitudes.

De manera que, la evaluación del bullying presenta dos perspectivas: una perspectiva individual, que debe recabar información sobre:

a) Aspectos personales de los sujetos implicados

b) Aspectos académicos

c) Nivel de indefensión/agresión y valoración conductual 
d) Aspectos relativos a sus relaciones interpersonales

e) Valoración del entorno familiar

f) Valoración del entorno escolar

Y una perspectiva grupal, donde se analice el conjunto del grupo de iguales para recabar información sobre:

a) Detección de alumnos directamente implicados y en situación de riesgo

b) Análisis sociométrico del grupo, configuración de los grupos de afinidad y lugar que ocupan los alumnos implicados

c) Valoración del grupo hacia los alumnos implicados

d) Forma, frecuencia y lugares habituales de agresión

e) Percepción de gravedad y/o seguridad en el centro

Los cuestionarios empleados en la evaluación del bullying, en general, pueden clasificarse en dos categorías: aquellos que pretenden el análisis de la incidencia del problema de manera anónima y general, indagando sobre la repercusión del fenómeno en cada sujeto considerado como observador, víctima o agresor (Olweus, 1993); y aquellos que pretenden una visión particular de la problemática a través de encuestas personalizadas o situaciones proyectivas. Entre los primeros, destaca por su amplia aplicación en Europa el cuestionario de Dan Olweus, a través de escalas tipo Likert y preguntas cerradas de respuesta múltiple, recaba información de forma anónima, sobre las características de las agresiones y sus protagonistas. Su corrección resulta muy laboriosa y además presenta una carencia que se revela como clave en este tipo de situaciones: el estudio de la estructura socio-afectiva del grupo. Otros instrumentos, tratando de evitar estos inconvenientes son demasiado concretos y reducidos, de manera que la información que aportan es muy limitada y se entienden como ayudas para sondeos previos, con lo cual se hace necesario establecer diferentes sesiones para completar la información, así ocurre con los elaborados por Smith y Sharp (1994). De la mayoría de ellos se han ido realizando adaptaciones, quizá en nuestro país la mas significativa sea la llevada a cabo por Ortega (1994) sobre el cuestionario de Olweus, titulado Cuestionario sobre actitudes intimidatorias y rechazo social entre escolares. Recientes estudios realizados en nuestro país proponen la aplicación de instrumentos específicos. Díaz-Aguado, Martínez y Martín (2004), elaboraron un Cuestionario de actitudes hacia la Diversidad y la Violencia (CADV), integrado por 71 elementos relacionados con: creencias hacia la diversidad y la violencia, disposi- 
ción hacia grupos minoritarios y disposición hacia el trabajo cooperativo. Este cuestionario está orientado hacia la evaluación de actitudes, pero ofrece elementos para el análisis de las relaciones socio-afectivas del grupo aula, además está indicado para alumnos de Educación Secundaria, por lo que puede ser considerado como un instrumento orientado a proporcionar información adicional al fenómeno bullying. Desde una perspectiva proyectiva, el equipo de Cristina del Barrio (Del Barrio, Almeida, Van der Meulen, Barrios, y Gutiérrez, 2003) a partir de intereses teóricos y metodológicos diseñó un instrumento ( $S C A N$-bullying) en forma de historieta gráfica que narra una relación de maltrato por abuso de poder en la escuela primaria, a través de entrevistas personales se pide a los alumnos que expresen su percepción sobre la naturaleza de la relación, atribuciones emocionales y estrategias de solución. Como sus autores indican, este instrumento persigue una finalidad metodológica para la investigación y por tanto resulta poco apropiado para su aplicación fuera de este contexto.

\section{El Test Bull-S. Características del material.}

El Test Bull-S es un cuestionario de aplicación colectiva, elaborado específicamente para la medida de la agresividad entre iguales en contextos escolares. Centrado en el análisis de la estructura interna del aula, y recabando información desde una doble perspectiva: la de los alumnos y la de sus profesores (formas A y P). En su planteamiento metodológico sigue la línea de la Sociometría, definida como "el estudio de la organización y evolución de los grupos y de la posición que en ellos ocupan los individuos prescindiendo de la estructura interna de cada individuo" (Moreno, 1972).

Dentro de esta compleja red encontramos que, los alumnos que se encuentran inmersos en la problemática de la agresión-victimización, suelen manifestar distorsiones en esta apreciación y generalmente son sujetos poco reconocidos, rechazados en la mayoría de los casos o cuando no, aislados. Además, el propio grupo genera un clima social tal que favorece esta dinámica al encubrir las situaciones de abuso y mostrar escasa preocupación por los víctimas (Cerezo, 1997; Gifford-Smith y Brownell, 2003; Sheridan, Buhs y Warnes, 2003).

El test Bull-S, compuesto por 15 ítems, está diseñado en torno a tres categorías de información: el estudio de la estructura interna del aula, a través de los criterios de aceptación rechazo, formada por cuatro ítems y mediante peer nomination. La dinámica bullying a través 
de seis ítems relativos a las características asociadas a los sujetos implicados y una tercera categoría relativa a aspectos situacionales con formato Likert.

La forma P (Profesores) pretende comprobar hasta qué punto el profesorado coincide con el alumnado en la apreciación del bullying.

\section{Ficha técnica.}

Nombre: TEST BULL-S. Medida de la agresividad entre escolares.

Formas: A (Alumnos) y P (Profesores)

Autora: Fuensanta Cerezo Ramírez

Administración: Forma A, colectiva. Forma P, individual

Duración: 25 a 30 minutos

Aplicación: Forma A para alumnado entre 7 y 16 años (Primaria y Secundaria). Forma P para profesores.

Significación: Análisis de las características socio-afectivas del grupo. Detección de implicados $\mathrm{y}$ aspectos situacionales en las relaciones de agresividad entre iguales.

Baremación: Sociograma del grupo. Posición sociométrica individual. Puntos de corte para valores significativos en las variables de agresión y de victimización. Frecuencias y porcentajes en aspectos situacionales.

Material: Ejemplar de cuestionario para la anotación en sus diferentes versiones A y P. Pizarra, lista de clase. Existe la posibilidad de tratamiento informatizado de los datos mediante CD-Rom.

\section{Validez y Fiabilidad de la prueba.}

Usando la variables relativas a Agresión y Victimización, el alfa de Cronbach para $\mathrm{N}=$ 322, fue de .73 y puntuó de manera similar en diferentes grupos de edad, sexo y aulas (los valores se situaron entre .69 y .75). Este valor aumentó considerablemente cuando fueron separados los ítems relativos a las conductas agresivas de los referidos a las conductas de victimización. Para los relativos a la agresión el alfa estimada fue de .82 y para los relativos a la victimización el alfa se situó en .83

La validación factorial entre los ítems, usando el análisis de componentes principales con rotación Varimax, explicó el $75.6 \%$ de la varianza total y mostró dos componentes polarizados: 
uno que agrupaba Cobardía, Víctimización y Tenerle Manía (con valores promedio de .86) y otro factor que agrupaba: Fortaleza física, Provocar y Agresividad (con valores promedio de .84). Uniendo las categorías sociométricas y bullying, el alfa de Cronbach fue de .68 y la varianza explicada se situó en el $76.8 \%$ del total, mostrando tres componentes: el primero, claramente asociado al sujeto víctima, agrupaba los ítems Rechazo, Cobardía, Victimización y Tenerle Manía (varianza promedio explicada .78), el segundo factor, asociado al bully, agrupa los ítems Fortaleza Física, Agresividad y Provocación (varianza promedio explicada .85), y un tercer factor que contiene solamente el ítem Aceptación, que podría asociarse a los no implicados, con una varianza explicada de -.96. Estos valores ponen de manifiesto la validez y fiabilidad de la prueba en unos límites muy aceptables.

\section{Análisis interpretativo del test Bull-S}

El análisis pormenorizado que a continuación presentamos se refiere a la Forma-A. Los elementos se han integrado en sus diferentes categorías.

CATEGORÍA I: Posición Sociométrica. La primera categoría agrupa a los ítems que indagan sobre la estructura socio-afectiva del grupo. Los ítems que componen esta categoría son: 1. Elegido, 2. Rechazado, 3. Ser elegido (expectativa) y 4. Ser rechazado (expectativa). Proporciona información referida a tres aspectos o dimensiones: la situación sociométrica, sobre la estructura informal del grupo y sobre el nivel de cohesión de sus miembros.

La situación sociométrica o estatus social de cada alumno en el grupo, evalúa el grado de aceptación de casa miembro del grupo bajo una triple perspectiva:

a) La relación de cada alumno con respecto al conjunto del grupo-aula, resultando valores que permiten clasificar a cada uno de ellos según el grado de aceptación o rechazo en las categorías de: líder, aislado, rechazado, etc. Asimismo, permite calcular el índice de popularidad y de antipatía. En general, informa del grado de acomodación del alumno al grupo de iguales.

b) Las expectativas sociales. Comparando el total de elecciones emitidas por el sujeto con el de las elecciones percibidas (esperadas), se llegan a establecer tres niveles de aceptación: Alta sociabilidad, cuando el total de elecciones emitidas es superior al que espera recibir. Baja sociabilidad, cuando espera recibir más elecciones de las que da y, por último, el sujeto equilibrado, cuando el total de las elecciones que espera recibir coincide con las que efectivamente recibe. 
c) Desde el análisis de estos ítems podemos avanzar en el conocimiento de la influencia que tienen algunos factores como el sexo y la edad en las elecciones o rechazos de los miembros y comprobar la repercusión que un líder o estrella tiene en el grupo.

Para la interpretación de los resultados tendremos en cuenta que:

1. Los valores MAYORES que el límite superior serán significativamente positivos. Estos alumnos serán pues: popular o rechazado, según la variable que hayamos trabajado.

2. Los valores MENORES que el límite inferior serán significativamente negativos. Estos alumnos serán: aislados, o no representativos.

3. Los valores comprendidos entre ambos límites no serán significativos.

Sobre la estructura del grupo, a través del concatenamiento de sus miembros que, en base a la atracción y el rechazo, forman una red sociométrica donde se aprecia la existencia de grupos y subgrupos: pandillas, parejas, triángulos, estrellas, etc. Y por último, permite conocer el Nivel de Cohesión o grado en que los miembros se sienten motivados a permanecer en el grupo. El análisis de esta dimensión en su conjunto, nos permite comprobar que, la mayoría de los sujetos se encuentran bien situados, es decir, sus expectativas coinciden con su estatus y su papel dentro del grupo. Sin embargo, los alumnos que se encuentran inmersos en la problemática de la agresión-victimización, suelen manifestar distorsiones en la percepción social $\mathrm{y}$, generalmente, son sujetos poco reconocidos, rechazados o cuando no, aislados (Cerezo, 2001a).

CATEGORÍA II: Dinámica Bullying: (Ítems 5-10). La segunda categoría informa de aspectos interpersonales en la relación agresión - victimización. Los ítems que la conforman son: 5. Fuerte, 6. Cobarde, 7. Agresivo, 8. Víctima, 9. Provoca, 10. Manía Su análisis nos informa sobre los elementos de la relación agresión-victimización entre los escolares, concretando: incidencia del problema y sujetos directamente implicados, la repercusión o apoyos que los sujetos implicados reciben del resto del grupo así como la percepción que de éstos tiene el grupo en cuanto a condiciones que favorecen esta dinámica de maltrato.

En cuanto a la repercusión que la dinámica Agresor-Víctima tiene en el grupo, el análisis abarca la perspectiva individual de los sujetos implicados, destacando la influencia del 
conjunto del grupo sobre este tipo de conductas, observa los apoyos que se otorgan al agresor o bully, y el grado de aceptación y reconocimiento de fortaleza. Por otro lado aprecia la incidencia de marcados afectos negativos sobre la víctima, que le llevan al aislamiento, y en definitiva, tanto sentir rechazo, como tenerle manía.

Desde una perspectiva grupal, el análisis muestra la red de relaciones interpersonales y así se aprecia la formación de pandillas o pequeños subgrupos en torno al bully.

El análisis de aspectos específicos de la dinámica bullying asociados al agresor y a la víctima nos proporcionan información sobre los sujetos que, en opinión de, al menos el 25\% del grupo, destacan o no- en cada una de ellas. Su estudio nos permite conocer el alcance de las situaciones de abuso, entendidas como una relación bipolar de agresividad y victimización entre los escolares. Las características que se asocian al perfil del agresor vienen marcadas por los ítems 5, 7 y 9 y las asociadas al de la víctima los ítems 6,8 y 10 . En ocasiones encontramos sujetos que puntúan de manera significativa en los ítems 9 y 10, éstos sujetos serán los llamados víctimas-provocadores. Otro aspecto que podemos detectar es la representación social que el grupo se forma sobre los sujetos involucrados en la dinámica agresor-víctima, valorando hasta qué punto justifica y/o aprueba estas situaciones.

CATEGORÍA III: Aspectos Situacionales. Está formada por los ítems 11. Forma, 12. Lugar, 13. Frecuencia, 14. Topografía, y 15. Seguridad. Recoge elementos concretos de las situaciones de abuso, explicitando la forma que adoptan, dónde suelen tener lugar, con qué frecuencia ocurren y el grado de gravedad que le atribuyen. Su análisis puede realizarse a dos niveles: individual, lo que permitirá conocer como se encuentra cada miembro del grupo y como valora esta problemática; y grupal a través de la conversión de las puntuaciones individuales en porcentajes, lo que informará sobre la visión del grupo en cuanto a formas mas habituales de agresión, frecuencia y lugares donde suelen ocurrir las agresiones. Asimismo nos dará el valor medio asignado al bullying (gravedad otorgada) y el nivel de seguridad percibida en el centro escolar.

\section{Propuestas de generalización y uso del test Bull-S.}

Cuando nos planteamos cómo intervenir en las situaciones de maltrato entre escolares, es necesario establecer unos elementos básicos en nuestro programa de manera que faciliten el 
conocimiento de la situación concreta en el centro escolar y propicien una postura antiagresión compartida ante la dinámica bullying. De forma explícita, el colectivo formado por profesores, padres y alumnos debe reflejar los principios y normas de conducta que regirán a todos por igual en la comunidad escolar. Se hace necesario dar cabida a todos los colectivos relacionados con el centro en la elaboración de una guía de actuación para prevenir y subsanar los problemas de convivencia, recogiendo en ella procedimientos y sistemas para prevenir y responder ante las situaciones de agresión. Estableciendo claramente los principios de acuerdo en términos de objetivos a alcanzar por todas las partes implicadas.

En el proceso de desarrollo de un programa de intervención se establecen cinco niveles consecutivos:

- Concienciación del problema en alumno, profesores y padres.

- Periodo de consultas y recogida de información.

- Confección del programa a través de asambleas de aula y acuerdos generales.

- Comunicación del plan a toda la comunidad y compromiso de cumplimiento.

- Puesta en funcionamiento, revisión y mantenimiento

El Bull-S puede servir como instrumento en la fase de recogida de información que contribuye eficazmente a la elaboración de pautas específicas para la intervención. Un esquema, a modo de ejemplo, puede encontrarse en el anexo.

El test Bull-S ha sido empleado en grupos de escolares de las diferentes comunidades autónomas, y traducido a las lenguas vernáculas de las mismas: euskera, catalán y gallego. Así mismo, su versión inglesa ha servido como instrumento para el análisis comparativo entre muestras de estudiantes españoles e ingleses (Cerezo y Ato, 2005). 


\section{Referencias}

AA.VV. (2000). Violencia escolar: El maltrato entre iguales en la educación secundaria. Oficina del Defensor del Pueblo. Madrid

Barudy, J. (1998). El dolor invisible. Una lectura ecosistémica del maltrato infantil. Barcelona: Paidós.

Cerezo, F. (1997). Conductas agresivas en la edad escolar. Madrid: Pirámide

Cerezo, F. (2000). El Test Bull-S. Instrumento para la evaluación de la agresividad entre escolares. Albor-Cohs. Madrid

Cerezo, F. (2001a). La violencia en las aulas. Análisis y Propuestas de Intervención. Madrid: Pirámide.

Cerezo, F. (2001b). Variables de personalidad asociadas a la dinámica bullying. (Agresores versus víctimas) en niños y niñas de 10 a 15 años. Anales de Psicología, 17 (1), 37-44.

Cerezo, F. (2002). El bullying y su relación con las actitudes de socialización en una muestra de adolescentes. Revista Electrónica Interuniversitaria de Formación del Profesorado, 5 (1). www.aufop.org/publica/reifp/02v5ni.asp

Cerezo, F. (2005). La violencia en la escuela en España. Ponencia presentada al IX Seminario Internacional sobre Biología y Sociología de la Violencia. Centro Reina Sofía para el Estudios de la Violencia. Valencia. Actas al congreso (203-209).

Cerezo, F y Ato, M. (2005) Bullying in Spanish and English pupils. A sociometric perspective using the Bull-S Questionnaire. Educational Psychology, 25(4), 353-368.

Cerezo, F.; Calvo, A. y Sánchez, C. (2004). Bullying y estatus social en el grupo-aula en una muestra de escolares. Comunicación presentada al IV Congreso Internacional de Psicología y Educación. Almería, 30 de Marzo al 2 de Abril de 2004. Actas del Congreso.

Del Barrio, C. Almeida, A, Van der Meulen, K.; Barrios, A. y Gutiérrez, H. (2003). Representaciones acerca del maltrato entre iguales, atribuciones emocionales y percepción de estrategias de cambio a partir de un instrumento narrativo: SCAN-Bullying. Infancia y Aprendizaje, 26 (1), 63-78.

Díaz-Aguado, M. J.; Martínez, R. y Martín, G. (2004). La violencia entre iguales en la escuela y en el ocio. Estudios comparativos e instrumentos de evaluación. Instituto de la Juventud. Madrid

Gifford-Smith, M.E. y Brownell, C.A. (2003). Childhood peer relationships: social acceptance, friendships, and peer networks. Journal of School Psychology, 41, 235-284. 
Leary, M. R.; Kowalski, R. M.; Smith, L. y Phillis, S. (2003). Teasing, rejection and violence: Case studies of school shootings. Aggressive Behavior, 29 (3) 202-214.

Moreno, J. L. (1972). Fundamentos de Sociometría. Buenos Aires: Paidós.

Olweus, D. (1993): Bullying al school: What we know and what we can do. Oxford. Blackwell. Trad. Conductas de acoso y amenaza entre escolares. Madrid. Morata 1998.

Ortega, R. (1994). Violencia interpersonal en los centros educativos de enseñanza secundaria. Un estudio sobre el maltrato y la intimidación entre compañeros. Revista de Educación, 304, 55-67

Roland, E. y Galloway, D. (2002). Classroom influences on bullying. Educational Research, 44, 299-312

Salmivalli, C. y Nieminen, E. (2002). Proactive and reactive agresión among school bulies, victims, and bully-victims. Aggressive Behavior, 28, 30-44.

Sheridan, S.M.; Buhs, E.S. y Warnes, E.D. (2003). Chilhood peer relationships in context. Journal of school Psychology, 41, 285-292.

Smith, P. K. y Sharp, S. (1994). Practical Approaches to Bullying. London: David Fulton Publishers.

Sutton, J.y Smith, P.K. (1999). Bullying as a group process: An adaptation of the participant role approach. Aggressive Behavior, 25, 97-111 
ANEXO.

\section{Esquema de informe a través del Bull-S.}

Centro Escolar: Situado en un barrio periférico de una ciudad mayor de 50.000 habitantes Aula: $6^{\circ}$ de Educación Primaria

Fecha: Marzo de 2005

\section{Análisis del estudio de las relaciones socio-afectivas entre el grupo de escolares.}

\section{Estructura y nivel de cohesión del grupo.}

Este grupo está formado por 30 alumnos de edades comprendidas entre los 11 y 12 años, 20 chicos y 10 chicas. Se observa 1 alumno popular, el n 17 (varón) y 4 aislados. Los alumnos rechazados son: el 15 y el 19 (varones) y el 1 (mujer). Con tendencia a ser rechazados encontramos al número 5 (varón). El nivel de cohesión es del 37.5, lo que indica una baja predisposición a sentirse miembros del grupo y por tanto desconexión entre sus miembros.

\section{Incidencia del Bullying:}

- Presencia y características: se observan 2 alumnos agresores, los números 15 y el 10, ambos varones; un alumno víctima, el 12; y un alumno víctima-provocador, el número 28.

- Nivel de Seguridad: en primer lugar se aprecia que la mayoría del grupo se encuentra seguro en el Centro, pero destaca que el 11.11\% confiese encontrarse Poco o Nada seguro en él, y el 22.22\% Regular. Lo que indica que un tercio de los escolares encuentran cierto riesgo en la seguridad del centro escolar.

- Importancia que los alumnos atribuyen a las agresiones: Poco o nada grave: 33.4\%; Regular: 41\%; Bastante grave: $18.6 \%$ y Muy Grave: 4\%.

- Frecuencia con la que ocurren: los porcentajes de las respuestas indican que las agresiones son percibidas por los alumnos de la siguiente manera:

Todos los días en el 77.8\%; 1-2 Veces por semana: 15\%; Rara vez: 7.5\% y Nunca el $3.5 \%$

- Formas de agresión mas frecuentes: en primer lugar, los alumnos señalan los insultos y amenazas (78\%); en segundo lugar, el rechazo (56\%); en tercer lugar Otras formas $(41 \%)$ entre las que indican pequeños hurtos y estropear el material de otros a propósito y, en cuarto lugar, el maltrato físico (45\%).

- Lugares donde suelen ocurrir las agresiones: en primer lugar, en el patio de recreo, señalado por el 74\%; después en el aula (30\%), seguido por Otros lugares (29.6\%) 
señalan los aseos, vestuarios y en la entrada del Colegio, y por último, en los pasi$\operatorname{llos}(37 \%)$.

\section{Alumnos en situación de riesgo}

a) Como sujetos receptores de bullying.

- Un alumno, sujeto número 12, varón de 11 años, aparece con marcada tendencia a ser víctima, así lo perciben el $71 \%$ de sus compañeros, le tienen manía el 52\% y es considerado cobarde por el $23 \%$ del grupo. Curiosamente sólo es elegido por el número 28 quién a su vez, es agresor y posiblemente lo utiliza como elemento de apoyo en sus acciones. El análisis sociométrico apunta que este alumno está aislado, no es sociable, puntúa muy bajo en expectativa de popularidad y es muy rechazado. El propio sujeto se reconoce víctima de maltrato y de ser objeto de manía por sus compañeros. Para él, la principal forma de agresión son los insultos y las amenazas, seguidas del rechazo, lo que ocurre todos los días en el patio de recreo y en el aula.

b) Como sujetos activos en bullying.

- El sujeto número 15 es varón, de 12 años, es considerado fuerte (34\%), agresivo (63\%) y provocador (75\%), opinión que corresponde con la suya propia en cuanto a fortaleza, pero no como agresor. En cuanto a su posición sociométrica aparece como aislado y rechazado, presenta pocas expectativas de ser aceptado y muchas de ser rechazado. Estos datos podrían apuntar a que se trata de agresor-reactivo. Su grupo de afinidad se limita al alumno número 3 el cual es considerado por la mayoría del grupo como uno de los sujetos mas fuertes de la clase y que, a su vez, se relaciona con los números 4 y 15 que también lo son. Esta circunstancia nos hace pensar que en este grupo existe un subgrupo de fuertesagresores.

- El sujeto número 10 es varón de 12 años, destaca como individuo agresivo (34\%) y provocador $(23 \%)$. En la variable aceptación aparece aislado y a la vez con cierta tendencia a ser rechazado. Su grupo de afinidad está formado, exclusivamente, por el número 15 . De nuevo se confirma la idea de la existencia de una pequeña red de relaciones en torno a las conductas de agresión y provocación.

c) Alumnos víctimas-provocadores.

- El sujeto número 28, también varón de 11 años, es considerado por sus compañeros y por él mismo como víctima (52\%), objeto de manía (41\%) y cobarde (45\%), junto con cierta 
tendencia a la agresión y la provocación, lo que le sitúa entre los dos tipos de conductas: agresión y victimización. Su grupo de afinidad está compuesto por el sujeto número 12 (recordemos que se trata de un alumno víctima). En cuanto a si posición sociométrica se encuentra aislado y rechazado. Posiblemente la tendencia a la agresividad forme parte de una estrategia de respuesta ante las situaciones conflictivas con sus compañeros, ya que su integración en el grupo es baja.

\section{Propuesta de intervención.}

Una vez analizados los resultados de la evaluación y comentados con los profesores y alumnos del grupo, pasamos a la elaboración del programa de intervención. Comenzando por delimitar las responsabilidades en el profesorado, por ejemplo llevando mayor vigilancia en los espacios de recreo y especialmente en el aula. Además, teniendo en cuenta que la imagen que el alumno tienen de sí mismo influye en su comportamiento social, los resultados del análisis sociométrico hacen necesario llevar a cabo actuaciones de desarrollo del autoconcepto y las habilidades sociales, punto donde el profesorado debe asumir gran responsabilidad, ya que su propia actitud servirá de pauta para el resto del grupo. Es importante que las estrategias y técnicas empleadas vayan dirigidas hacia el cambio de actitudes de víctimas y agresores, y sobre la actitud del grupo hacia ellos. Se hace necesario asimismo, la implicación, compromiso y colaboración entre familia y escuela.

El programa está estructurado en cuatro niveles, en cada uno de ellos se concretan las estrategias que se consideran mas apropiadas y efectivas. Estos niveles son:

\subsection{Institucional.}

El Centro escolar debe asumir como tarea primordial la mejora del clima educativo en sus aulas, para ello, no solo posibilitará, sino que potenciará estrategias como las siguientes:

- Reuniones periódicas del profesorado donde se analicen las situaciones detectadas y se propongan estrategias de intervención educativas.

- Gestionar la comunicación con los alumnos implicados y sus familias.

- Programar actividades específicas como mesas redondas, cineforum, etc., en las que participen alumnos, profesores, padres y expertos, para asesorar, comentar, poner ideas en común y proponer estrategias de intervención educativas.

- Facilitar la participación del profesorado en actividades extraescolares de tipo lúdico, para superar la simple relación profesor/alumno, fomentando la comunicación y las relaciones personales. 
- Solicitar al profesorado una mayor vigilancia en los espacios comunes.

- Facilitar la comunicación con las familias.

- Estar abierto a cualquier iniciativa que, desde la comunidad escolar, pueda favorecer la convivencia.

\subsection{El grupo-aula.}

En nuestro caso, teniendo en cuenta que los resultados del cuestionario han revelado que el índice de cohesión de los miembros del grupo es bajo y se observan bastantes sujetos aislados, nuestra propuesta irá encaminada a incrementar el sentimiento de grupo así como a mitigar la dinámica bullying.

Antes de elaborar un plan concreto indagaremos en el grupo qué se podría hacer para integrar a los sujetos aislados, agresores y víctimas. Hay que llegar a romper el silencio y la actitud de aceptación de la situación conflictiva y de apoyo al agresor.

Las técnicas que proponemos para incluir en las sesiones de tutoría se articulan en torno a los Grupos de Discusión, el Rol-play o dramatización, la Resolución de conflictos y las Técnicas de aprendizaje cooperativo

\subsection{Los alumnos de riesgo.}

Se elabora un programa específico para cada uno de los alumnos que se encuentran implicados en la dinámica bullying: bullies, víctimas y víctimas/provocadores o con cierta tendencia a la agresividad.

Como quedó de manifiesto en la evaluación, en el grupo se encuentran dos alumnos que sufren claramente maltrato por algunos de sus compañeros (números 12 y 28) y a su vez, el número 28 puede considerarse víctima/provocador. Asimismo, se detectó la presencia de dos alumnos agresores (números 10 y15). Antes de empezar a trabajar con cada uno de estos alumnos, evaluaremos con mayor precisión las características de su situación tanto académica como personal y familiar. Esta información será recabada a través del profesor-tutor, y en su caso con la colaboración del orientador del Centro. Para ello será necesario mantener una serie de entrevistas personales con los profesores, los alumnos y los padres. En general debemos tener en cuenta los siguientes aspectos:

1) Aspectos a trabajar con las víctimas: En las entrevistas previas trabajamos la expresión de sentimientos e indagamos en los posibles motivos a los que ellos atribuyen estas situaciones. Como estrategias específicas plantearemos: mejorar el autoconcepto, el entrenamiento en 
habilidades para la resolución de conflictos; la expresión de sentimientos; estrategias de estudio y concentración y estrategias para desarrollar estilos atribucionales positivos.

2) Aspectos a trabajar con los agresores. A la hora de trabajar con estos alumnos debemos tener claro que nuestro objetivo prioritario es que dejen de agredir, pero a la vez, que se encuentren a gusto en el Centro Educativo. Las estrategias pues, deben plantearse a tres niveles:

- Las conductas agresivas no son admisibles en el código de conducta prosocial.

- La necesidad de asumir modelos de conducta apropiados

- Cambio de actitudes y comportamientos.

En las entrevistas personales previas trataremos de precisar los motivos de su comportamiento destacando aquellos elementos que puedan clarificar su actitud ante la agresión.

Las estrategias de intervención propuestas abarcan los siguientes aspectos: Entrenamiento asertivo; autoobservación, autoregistro y autorregulación; solución de problemas; relajación y técnicas de estudio.

3) Aspectos a trabajar con el víctima-provocador.- Tomando aspectos de los modelos anteriores en los que incluiremos tareas específicas para la mejora del autoconcepto y el entrenamiento en habilidades sociales.

4) Programa de trabajo conjunto para los alumnos implicados.- Para consolidar un cambio de actitudes es necesario que los sujetos implicados colaboren en la búsqueda de soluciones al problema conjuntamente y se impliquen en la mejora de la relación. Este es el objetivo que perseguimos con este programa. La estrategias empleadas se basan en el modelo de la mediación, es decir, llegar a tomar conciencia de los propios sentimientos y de los del adversario, para que, de esta manera surjan lazos de respeto y comunicación. Para llevar a efecto se deberán planificar sesiones de trabajo conjunto con el orientador. En ellas se concretarán espacios y tiempos, así como las revisiones al plan de ejecución y evaluación de resultados. 
Violencia y victimización entre iguales. El bullying: estrategias de identificación y elementos para la intervención a través del Test Bull-S

[Página en blanco por razones de paginación] 